Original Paper http://ajol.info/index.php/ijbcs http://indexmedicus.afro.who.int

\title{
Perception endogène de l'influence des changements climatiques sur la pêche dans la basse vallée de l'Ouémé (Sud Bénin)
}

\author{
A.H. ATTINGLI ${ }^{1 *}$, E.W. VISSIN ${ }^{2}$, S. AHOUANSOU-MONTCHO ${ }^{1}$, L. H. ZINSOU ${ }^{1}$ et \\ P.A. LALEYE ${ }^{1}$ \\ ${ }^{1}$ Laboratoire d'Hydrobiologie et d'Aquaculture (LHA), Faculté des Sciences \\ Agronomiques (FSA), Université d'Abomey -Calavi. 01 BP 526, Cotonou, Bénin. \\ ${ }^{2}$ Laboratoire Pierre PAGNEY Climat, Eau, Ecosystème et Développement, (LACEEDE), \\ Université d'Abomey - Calavi. 01 BP 526, Cotonou, Bénin. \\ *Auteur correspondant ; E-mail: hermasattingli@yahoo.fr
}

\section{RESUME}

Les perceptions endogènes des pêcheurs de l'influence des changements climatiques sur la pêche dans la basse vallée de l'Ouémé ont été étudiées. Ces perceptions ont été recueillies à l'aide d'un questionnaire administré à 266 pêcheurs d'au moins 20 ans d'expérience, exerçant la pêche dans Aguégués, Dangbo, Adjohoun et Bonou. Il s'agit des autochtones dont 43,98\% de pêcheur-agriculteurs 35,71\% d'agriculteurpêcheurs, 18,42\% de pêcheurs reconvertis en exploitants de sable fluvial, 1,13\% reconvertis en transport fluvial et $0,75 \%$ reconvertis en trafic d'essence. La modélisation des tendances évolutives (1971 et 2013) de la pluviométrie annuelle, de la température et de la lame d'eau écoulée a été réalisée avec le logiciel Minitab 1.4., en utilisant l'analyse des séries chronologiques. Une analyse factorielle des correspondances (AFC) a servi à décrire les déterminants des perceptions. Les pêcheurs ont signalé une augmentation de la température et une diminution de la pluviométrie. Ils ont fait cas des crues sévères, des inondations persistantes et de la baisse des rendements des captures. Leurs perceptions sont corroborées par l'analyse des données hydrométéorologiques et les statistiques de pêche. Une régression logistique binaire avec le model Proc logit du logiciel SAS 9.2 a montré que le sexe influence significativement $(\mathrm{p}<0,001)$ au seuil de $5 \%$ la perception des pêcheurs, de même que leur appartenance religieuse $(\mathrm{p}<0,1)$ au seuil de et $10 \%$.

(C) 2016 International Formulae Group. All rights reserved.

Mots clés : Perceptions des pêcheurs, changements climatiques, basse vallée de l'Ouémé.

\section{Endogenous perceptions of the influence of climate change on fisheries in the lower valley of Ouémé (South Benin)}

\begin{abstract}
This study aims to analyze endogenous perceptions of fishermen relative to the influence of climate change on fisheries in the lower valley of Ouémé. Data on socio-professional characteristics of the fishermen
\end{abstract}


were collected. Their perceptions were collected using a questionnaire administered to 266 fishermen of at least 20 years old. Those fishermen pursued fishing in Aguégués, Dangbo, Adjohoun and Bonou. The modeling of evolutionary trends (1971 to 2013) of annual rainfall, temperature and runoff was done with Minitab software 1.4 using time series analysis. A correspondence factor analysis (CFA) was used to describe the determinants of fishermen's perceptions. The results showed that fishermen were native. $43.98 \%$ of them were fisherfarmers, $35.71 \%$ were farmer-fishermen, $18.42 \%$ were fishermen reconverted into fluvial sands operators, $1.13 \%$ were converted fluvial carriers, and $0.75 \%$ converted into gasoline traffic. The fishermen reported an increased temperature and decreased rainfall. They have also reported severe floods, persistent flooding and declining catches yields. Their perceptions were supported by the analysis of hydrometeorological data and fisheries statistics. A binary logistic regression performed with the Proc logit model of SAS 9.2 software showed that sex significantly influenced $(\mathrm{p}<0.001)$ the perception of the fishermen at $5 \%$. Also religious affiliation determined the perception of the fishermen $(\mathrm{p}<0.1)$ at the $10 \%$ threshold.

(c) 2016 International Formulae Group. All rights reserved.

Keywords: Fisher's perceptions, climate change, low valley of Ouémé.

\section{INTRODUCTION.}

Les changements climatiques constituent une question primordiale en raison de leurs conséquences sur la biodiversité et les sociétés humaines (Gnanglè et al., 2012). En Afrique, le passage des conditions humides de 1950 et 1960 aux conditions sèches de 1970 à 1990 a été traduit par une variation sensible des systèmes hydroclimatiques (Vodounou et al., 2011). L'impact des changements climatiques paraît ainsi très sévère en Afrique de l'Ouest où la plupart des pays sont moins aptes à s'adapter (Sonneveld et al., 2012). La vulnérabilité des pays et de leurs populations aux changements climatiques demeure une grande préoccupation au point de susciter un intérêt scientifique considérable au cours des dernières décennies, avec des appels à une augmentation $\mathrm{du}$ financement pour l'adaptation (Patt et al., 2010).

Dans le contexte ou l'existence d'impacts néfaste du changement climatique sur la production agricole en Afrique subsaharienne est maintenant solidement établie (Gbaguidi et al., 2015), pour définir les stratégies adéquates face aux changements climatiques, il est nécessaire de recueillir la perception des acteurs sur ses manifestations. O'Brien et al. (2010) fait observer que l'adoption des mesures d'adaptation est en partie déterminée ou limitée par la perception que les populations rurales ont des changements climatiques. En Afrique de l'ouest, notamment en Côte d'Ivoire des travaux sont effectués de façon globale sur les perceptions paysannes des changements climatiques (Brou et al., 2005) et au Bénin (Gnanglè et al., 2012; Loko et al., 2013). Toutefois, les perceptions des pêcheurs face aux changements climatiques relatives à la conservation de la biodiversité aquatique en général et des poissons en particulier restent à étudier au regard de sa complexité socioéconomique.

Au Bénin, les zones agroécologiques sont très vulnérables aux changements climatiques (PANA-Bénin, 2008). Notamment la zone agroécologique 8 dite "des pêcheries" (INRAB, 1995) abritant la basse vallée de l'Ouémé. En effet, elle .est confrontée à un régime pluviométrique marqué par une forte instabilité, caractéristique des régions du sud Bénin (Yabi et al., 2009) et par une forte installation du régime unimodal (Ogouwalé et Boko, 2007). Les perturbations climatiques apparaissent ainsi comme une menace évidente pour la biodiversité aquatique de la basse vallée de l'Ouémé où le rendement des principaux engins est en baisse depuis quelques décennies (Lalèyè et al., 2004). Eu égard à ce constat, Il parait nécessaire d'intégrer la perception des pêcheurs de la 
basse vallée de l'Ouémé de l'impact des changements climatiques sur la pêche afin d'y apporter efficacement des solutions.

Cette étude est une contribution à l'évaluation de la perception endogène des pêcheurs sur les changements climatiques et son impact sur la pêche. Elle vise à documenter les perceptions des pêcheurs sur les changements climatiques; de comparer ces perceptions aux données hydroclimatiques et de pêche ; d'identifier les facteurs influençant la perception des changements climatiques par les pêcheurs ainsi que ses impacts sur les activités de pêche.

\section{MATÉRIEL ET MÉTHODES}

\section{Présentation de la zone d'étude}

La basse vallée de l'Ouémé comporte de vastes plaines d'inondation dont les caractéristiques écologiques favorisent une importante colonisation par les poissons (Lalèyè et al., 2004). Elle est intégrée à la partie inférieure du fleuve Ouémé, long de $510 \mathrm{Km}$, formant le plus grand bassin fluvial $\left(50000 \mathrm{~km}^{2}\right)$ du Bénin. Etalée entre6 ${ }^{\circ} 24^{\prime}-$ $6^{\circ} 52^{\prime} \mathrm{N}$, et $2^{\circ} 24^{\prime}-2^{\circ} 38^{\prime}$ E, elle recouvre plus de $9000 \mathrm{Km}^{2}$ et son régime hydrologique est tributaire du climat soudanien (Nord-Bénin). Les communes des Aguégués, de Dangbo, d'Adjohoun et de Bonou sont intégrées à la basse vallée de l'Ouémé.

Le choix des zones de pêcherie a été fait de façon raisonnée. Les critères utilisés sont relatifs à la proximité des agglomérations des pêcheurs, l'accessibilité des zones, l'existence d'un débarcadère d'au moins 10 pirogues et l'exercice effectif de l'activité de pêche. Au total, seize (16) zones de pêcheries et leurs périphéries (rayon de $500 \mathrm{~m}$ ) ont été considérées d'amont en aval dans la basse vallée du fleuve Ouémé (Figure 1).

\section{Méthodes et outils de collecte des données}

L'enquête a été conduite de mai 2014 à avril 2015. Elle a permis de recueillir au moyen d'un questionnaire les avis des pêcheurs sur l'évolution des activités de pêche dans le temps en tenant compte des effets induits par les changements climatiques. Avant l'enquête proprement dite, une étude exploratoire a été conduite dans la zone d'étude.

A partir des valeurs $p$ issues des résultats de la phase exploratoire de l'étude, la taille de l'échantillon dans chacune des 16 pêcheries (localités) des 4 communes a été définie par une approximation normale de la distribution binomiale selon Dagnelie (1998) suivant la formule: $\mathrm{N}=\left[\left(\mathrm{U}_{1-\alpha / 2}\right)^{2} \times \mathrm{p}(1-\mathrm{p})\right] / \mathrm{d}^{2}$; $\mathrm{U}=$ valeur de la variable aléatoire normale pour la valeur de probabilité de $1-\alpha / 2 ; \mathrm{p}=$ probabilité de $1-\alpha / 2, \alpha=$ risque d'erreur, $d=$ marge d'erreur d'estimation. Pour $\alpha=5 \%$, la probabilité $\mathrm{p}=1-\alpha / 2=0,975$ et $\mathrm{U}_{1-\alpha / 2}=1,96$. p est la proportion de pêcheurs ayant au moins 20 ans d'expérience dans le métier et la marge d'erreur d'estimation, retenue à $8 \%$ dans cette étude (Assogbadjo et al., 2011). Ainsi, 96, 77, 44 et 49 , pêcheurs ont été enquêtés respectivement à Aguégués, Dangbo, Adjohoun et Bonou. Les informations quantitatives collectées sont relatives aux caractéristiques socioprofessionnelles des pêcheurs (sexe, religion, situation matrimoniale, activité principale et secondaire...), Les types de données qualitatives collectées sont les perceptions des pêcheurs sur les impacts des changements climatiques. Pour la nécessité de comparaison des déclarations des pêcheurs et informations scientifiques, les données climatologiques de la zone d'étude (pluviométrie (mm), températures $\left({ }^{\circ} \mathrm{C}\right)$ sur la période de 1971 à 2013, 43 années, ont été recueillies auprès de l'Agence pour la Sécurité de la Navigation Aérienne en Afrique et à Madagascar (ASECNA, 1971-2013). Les données statistiques hydrométriques (lame d'eau écoulée en $\mathrm{mm}$ ) sur la même période ont été recueillies auprès des Services de l'Hydrologie de la Direction Générale de l'Eau à Cotonou (DG-Eau, 1971-2013). Les 
statistiques de pêche de quatre familles de poissons-chats les plus représentées (Mochokidae, Clariidae, Schilbeidae et Claroteidae) ont été obtenues auprès de la Direction des Ressources Halieutiques (ex Direction des Pêches) (DP, 1987-2000).

\section{Traitements et analyse de données}

Les différentes informations issues des enquêtes socioprofessionnelles ont été présentées sous forme de graphes avec les pourcentages de réponses. La modélisation de la tendance évolutive entre 1971 et 2013 de la pluviométrie annuelle, la température et la lame d'eau écoulée a été faite avec le logiciel Minitab 1.4., en utilisant l'analyse des séries chronologiques, plus précisément l'analyse des tendances (Bowerman et O'Connell, 1993). L'ajustement linéaire des tendances observées est aussi effectué en tenant compte de certains paramètres de précision comme l'erreur moyenne absolue en pourcentage (MAPE), la déviation moyenne absolue (MAD) et la déviation moyenne quadratique (MSD) des séries estimées par rapport aux valeurs réelles observées. Une faible valeur de ces paramètres climatiques indique un bon ajustement de la série chronologique selon les mêmes auteurs

Afin d'identifier les déterminants des perceptions des pêcheurs au regard de la variabilité climatique, le model Proc logit du logiciel SAS version 9.2 a été utilisé pour la régression logistique binaire. Elle a porté sur les variables dépendantes telles que la perception climatique. Pour la variable perception climatique (oui ou non), les variables indépendantes sont: niveau d'instruction, sexe, âge, expérience professionnelle et la religion pratiquée. A cet effet, le test Khi2 de Pearson a été utilisé pour apprécier les déterminants qui influent sur chaque variable la réponse perception. De plus, des tableaux ont été soumis à une Analyse Factorielle des Correspondances afin de décrire les relations existantes entre la perception des pêcheurs et les facteurs qui favorisent l'ampleur des variabilités climatiques dans les 4 communes.

\section{RESULTATS}

\section{Caractéristiques socioprofessionnelles des acteurs de pêche enquêtés}

Le Tableau 1 présente les caractéristiques socio-professionnelles des pêcheurs enquêtés. L'âge de la majorité des pêcheurs enquêtés $(87,22 \%)$ se situe entre 30 et 60 ans, et ces pêcheurs sont par ailleurs mariés (98,12\%). 96,61\% d'entre eux ont entre 20 à 40 ans d'expérience dans la pratique de la pêche. La contribution des activités de pêche dans le revenu du ménage depuis quelques années n'est pas importante selon la majorité. Ces pêcheurs sont des autochtones, dont 43,98\% de pêcheursagriculteurs, 35,71\% d'agriculteurs-pêcheurs, $18,42 \%$ de pêcheurs reconvertis en exploitants de sable fluvial, $1,13 \%$ reconvertis en transport fluvial et $0,75 \%$ reconvertis en trafic d'essence (Figure 2). Ils considèrent la pêche comme une activité génératrice de revenus en déclin dans leur terroir.

\section{Perception des pêcheurs des changements climatiques et comparaison avec les données hydroclimatiques}

Les pêcheurs ont indiqué 4 conditions idéales qui favorisaient la pêche: une pluviosité bonne et stable $(48,87 \%$ des enquêtés), un bon niveau quasi permanent de l'eau même en période d'étiage $(36,47 \%$ des enquêtés), une température moyenne $(6,77 \%$ des enquêtés), et un ensoleillement moyen (4, $89 \%$ des enquêtés). Par ailleurs, $73 \%$ des enquêtés ont indiqué qu'il y a plus de trente ans, les conditions hydroclimatiques étaient favorables à la pêche alors que de nos jours, il y a de façon notable, des modifications perceptibles au niveau de la pluviométrie et de la température surtout en période d'étiage. Les 
variabilités sont signalées à 7 niveaux (Figure 3). Ainsi, le retard des pluies, l'arrêt précoce des pluies, la diminution de la quantité des pluies sont les plus importants et représentent à eux seuls $92 \%$ des réponses. Les pêcheurs ont signalé une élévation de la température $(63,16 \%)$, une augmentation de la durée de l'insolation (20,30\%), un assèchement du cours d'eau par endroits $(6,77 \%)$ et un début précoce de la sècheresse 6,02\% (Figure 4).

L'analyse de régression logistique binaire a révélé que le sexe $(p<0,001)$ influence significativement la perception des pêcheurs au seuil de 5\%, tandis que l'appartenance religieuse $(\mathrm{p}<0,1) \quad$ est déterminante au seuil de $10 \%$ (Tableau 2). La variation temporelle entre 1971 et 2013 de la température minimale et maximale (Figures 5 et 6) indique globalement une tendance évolutive avec un taux de croissance moyen de $0,02{ }^{\circ} \mathrm{C} / \mathrm{an}$ selon un ajustement linéaire. Cet ajustement linéaire est très significatif au regard des paramètres de précision MAPE, MAD et MSD qui sont tous faibles. Le minimum le plus élevé est observé en 2010 avec une moyenne de $25,7{ }^{\circ} \mathrm{C}$ alors que les maxima les plus élevés sont observés en 1973 et 2008 avec des moyennes respectives de 31 ${ }^{\circ} \mathrm{C}$ et $30,9{ }^{\circ} \mathrm{C}$. De même, la tendance évolutive de la lame d'eau écoulée présente une allure linéaire avec un taux de croissance moyen de 1,67 mm/an. Cette tendance est relativement significative au regard des paramètres de précision MAPE, MAD et MSD qui sont tous relativement élevés (Figure 7). Toutefois, on note une chute continue de la lame d'eau écoulée à partir des années 2008.

S'agissant de la pluviométrie (Figure 8), Les valeurs très élevées de MAPE, MAD, MSD traduisent une faible significativité de la tendance linéaire simulée et une fluctuation très prononcée de la pluviosité dans la période allant de 1971 à 2013. La superposition des données hydroclimatiques avec les indications

des pêcheurs montre qu'il y a de nombreuses concordances. En effet, les pêcheurs ont bien rapporté une diminution de la quantité de pluie, une augmentation des températures, une diminution des lames d'eau écoulée pour exprimer l'ampleur des variabilités climatiques dans la basse vallée de l'Ouémé.

\section{Impact des changements climatiques sur les pêches}

Les pêcheurs ont signalé les impacts négatifs de ces modifications sur la pêche avec l'appauvrissement en ressources halieutiques des plans d'eau, l'attaque des poissons par des parasites avec l'augmentation de la température de l'eau, la prolifération des plantes ichtyotoxiques, puis la rareté des espèces économiquement rentables dans les captures de pêches (Figure 9). Ce point de vue des pêcheurs est renforcé par la Figure 10. En effet, ces informations sont superposables avec les chiffres obtenus sur l'évolution des captures des quatre principales familles de poissons-chats (Claroteidae, Clariidae, Mochokidae et Schilbeidae). Globalement les captures de ces principales familles de poissons sont en nette régression.

$\begin{aligned} & \text { Actions favorisant l'ampleur des } \\ & \text { changements climatiques }\end{aligned}$
L'analyse factorielle des
correspondances (AFC) effectuée pour
analyser les relations entre communes a révélé
que deux composantes principales expliquent
$98.09 \%$ des actions qui favorisent l'ampleur
des changements climatiques. Ainsi, les
pêcheurs d'Adjohoun pensent que
l'exploitation effrénée du sable fluvial peut
être l'une des raisons favorables. A Dangbo,
les pêcheurs lient cet état de chose à la
déforestation avancée tandis qu'à Bonou, les
pêcheurs ont mis l'accent sur l'enfreinte aux
mœurs endogènes et traditionnelles (Figure
11).




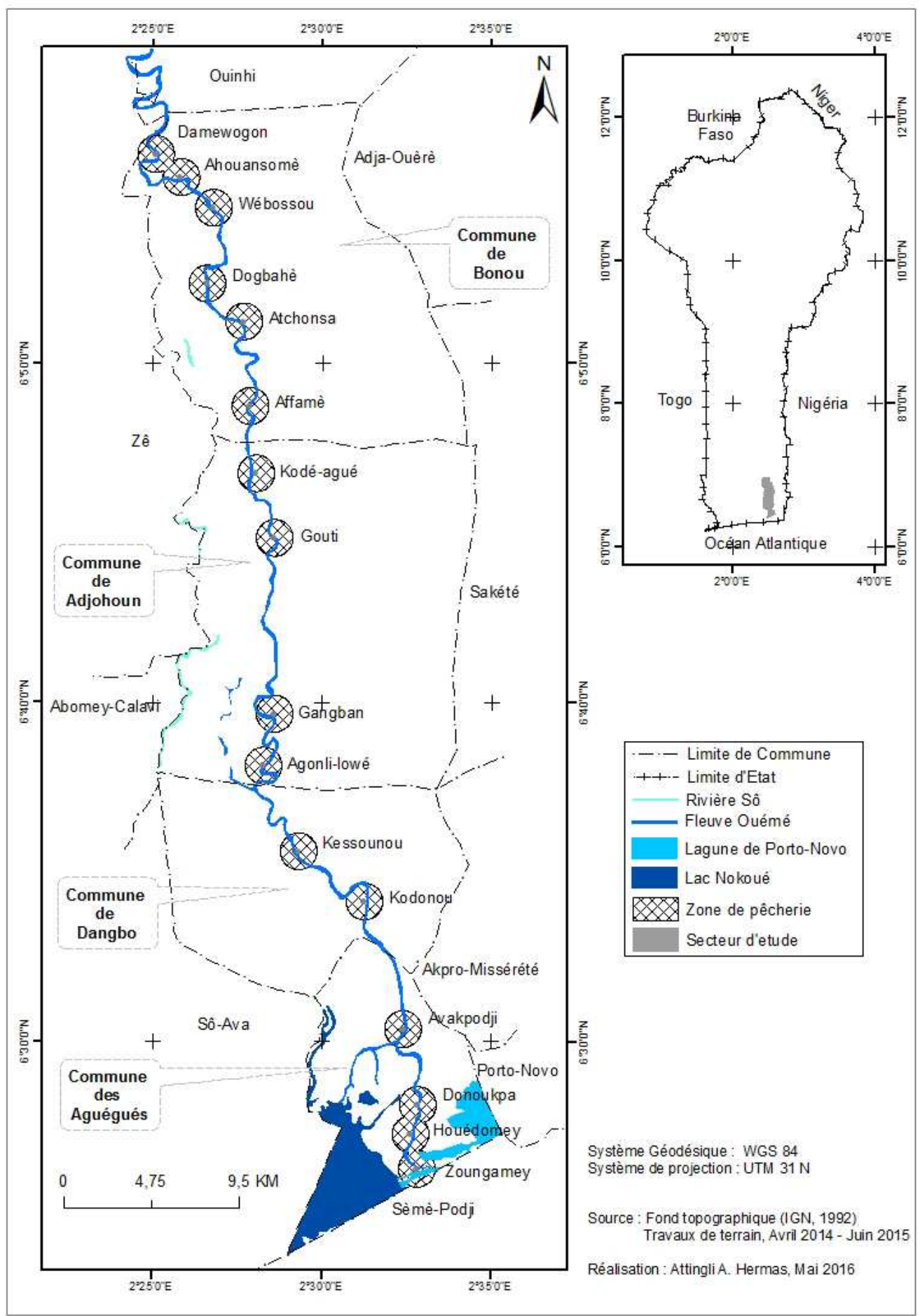

Figure 1: Localisation géographique des pêcheries dans la basse vallée de l'Ouémé. Les 16 pêcheries enquêtées sont reparties le long de la basse vallée de l'Ouémé entre 4 communes. (Aguégués, Dangbo, Adjohoun et Bonou). 


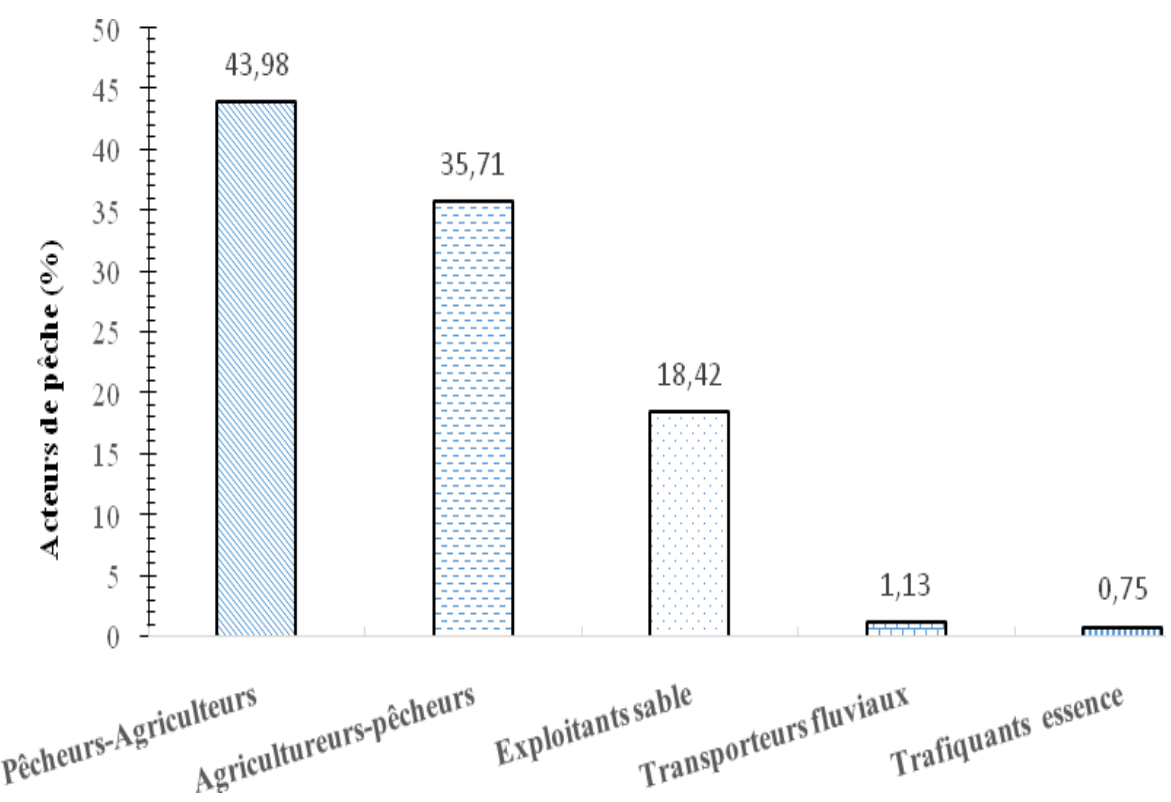

Figure 2: Caractéristiques socio-professionnelles des acteurs de pêche dans la basse vallée de l'Ouémé.

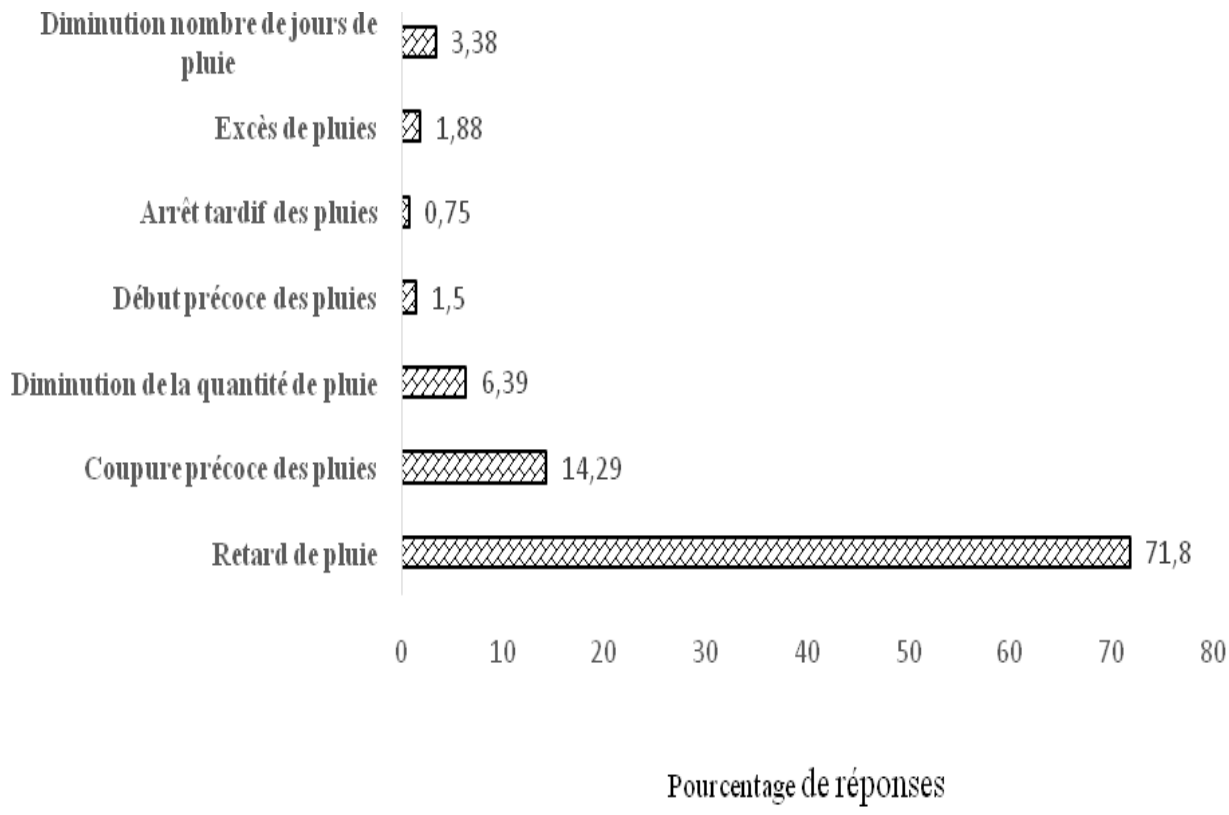

Figure 3: Perceptions pêcheurs des changements climatiques liés à la pluviométrie. 
Assèch em ent du cours du fleuve par endroit

Sèch eresse prolongée

Réduction de la fraich emr'nocturne

2,63

Débutprécoce de la sècheresse

Elévation de la température

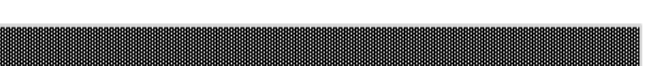

\section{Pourcentage de rép onses}

Figure 4: Perceptions pêcheurs des changements climatiques liés à la température.

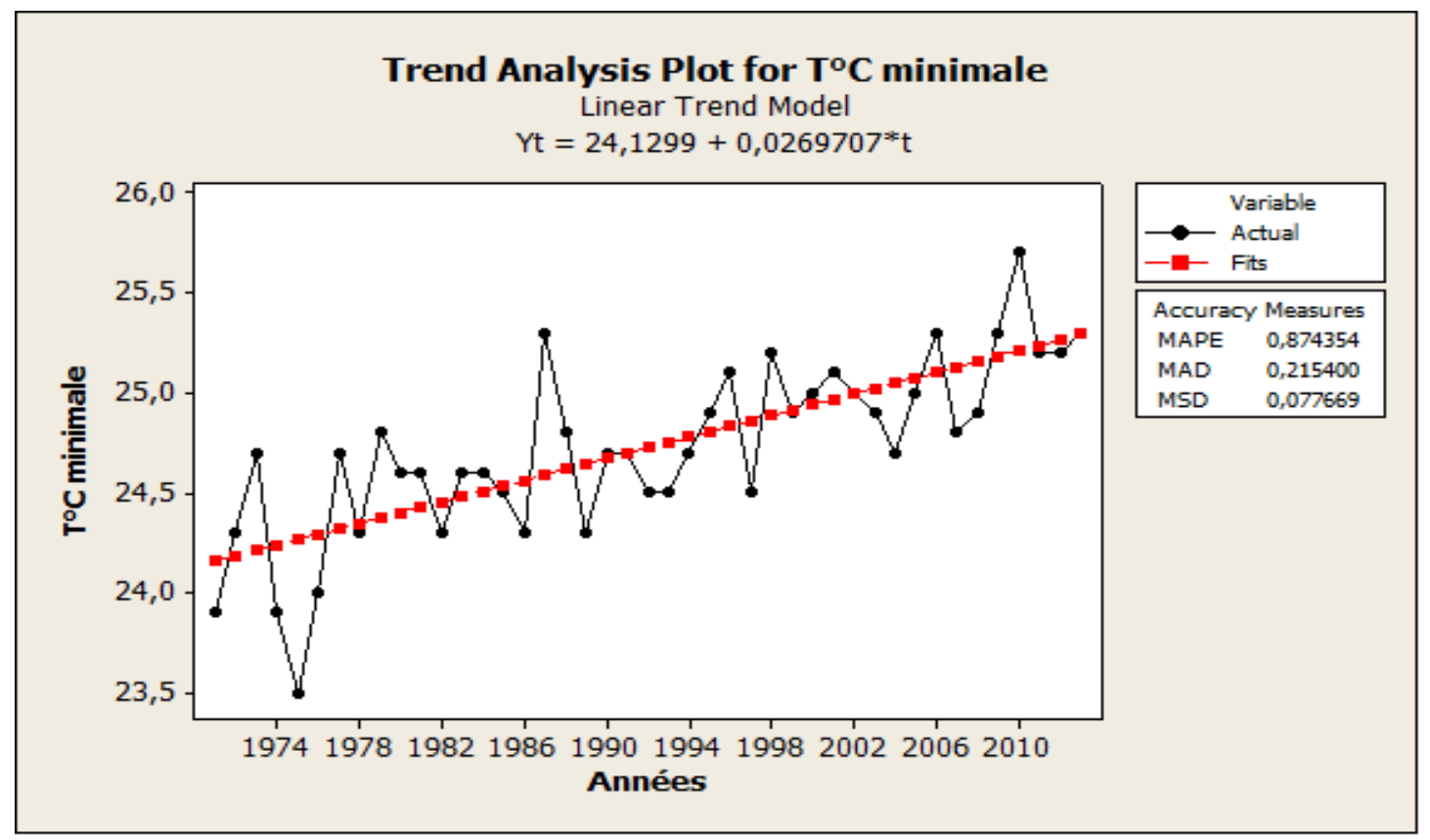

Figure 5: Courbe de tendance de l'évolution annuelle de la température minimale dans la basse vallée de l'Ouémé. MAPE : erreur moyenne absolue en pourcentage ; MAD : déviation moyenne absolue ; MSD : déviation moyenne quadratique. 


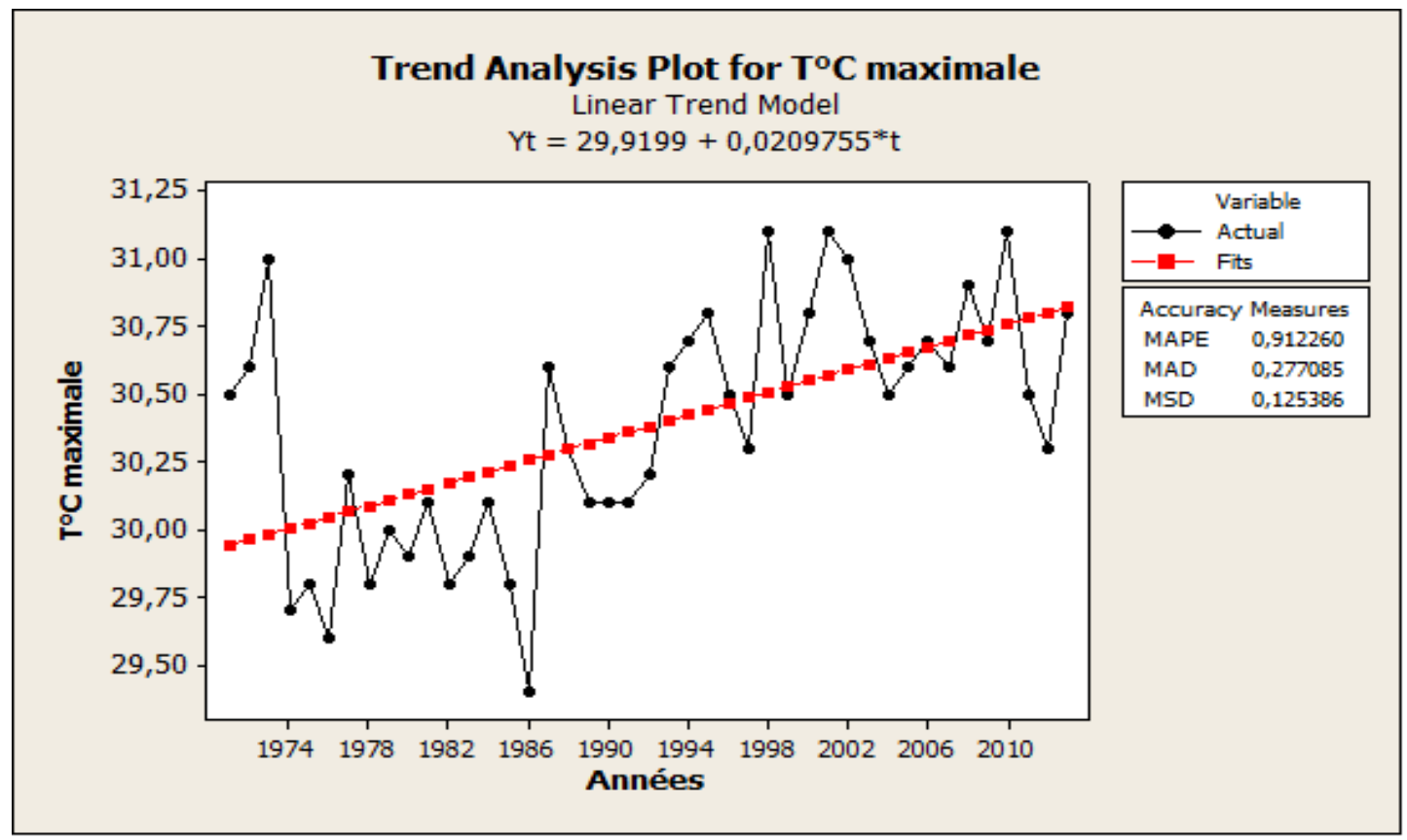

Figure 6: Courbe de tendance de l'évolution annuelle de la température maximale dans la basse vallée de l'Ouémé. MAPE : erreur moyenne absolue en pourcentage ; MAD : déviation moyenne absolue ; MSD : déviation moyenne quadratique.

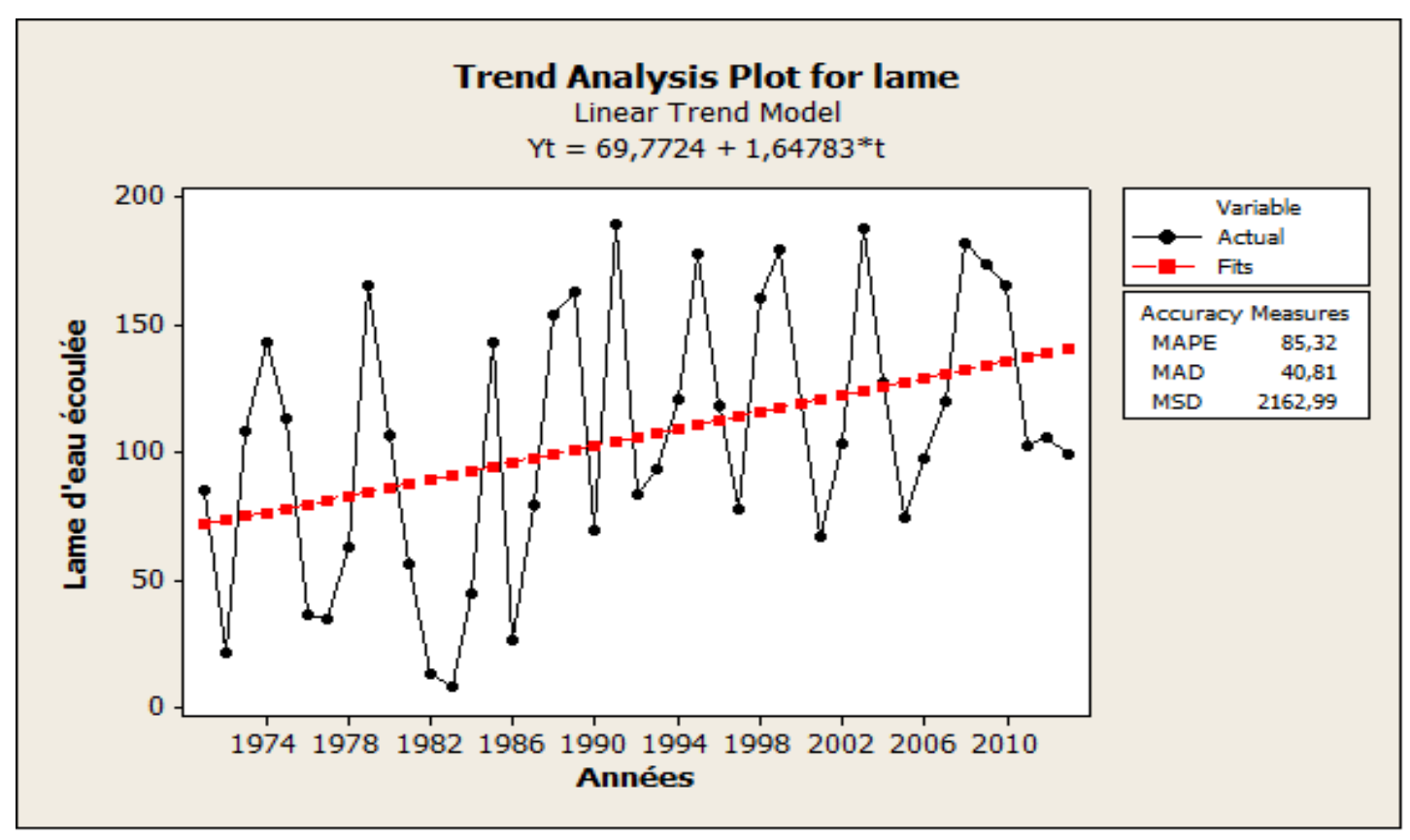

Figure 7: Courbe de tendance de la variation interannuelle de la lame d'eau écoulée dans la basse vallée (1971-2013). MAPE : erreur moyenne absolue en pourcentage ; MAD : déviation moyenne absolue; MSD : déviation moyenne quadratique. 


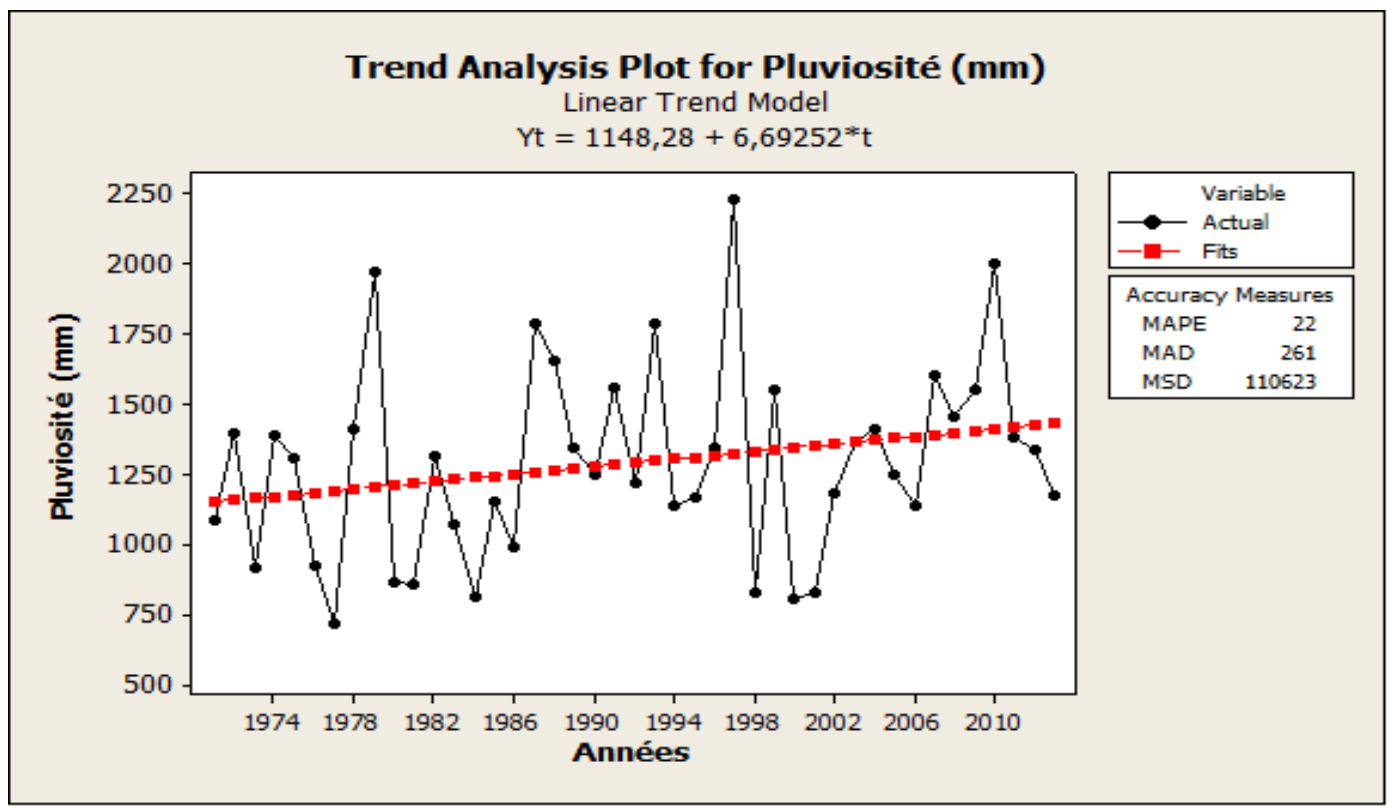

Figure 8: Courbe de tendance de l'évolution interannuelle des précipitations dans la basse vallée de l'Ouémé (1971-2013).

MAPE : erreur moyenne absolue en pourcentage ; MAD : déviation moyenne absolue ; MSD : déviation moyenne quadratique.

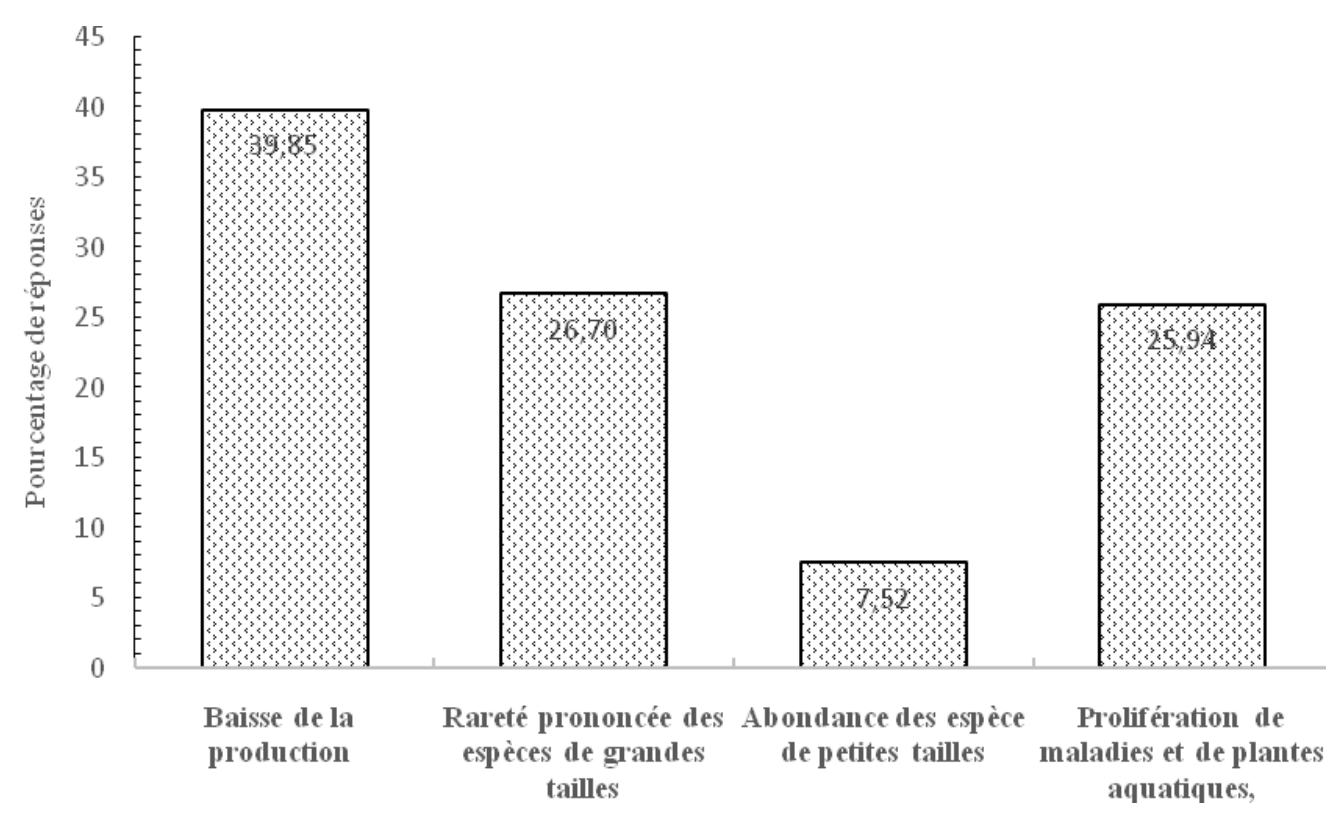

Figure 9: Perceptions des pêcheurs de l'impact des changements climatiques sur les pêches. 


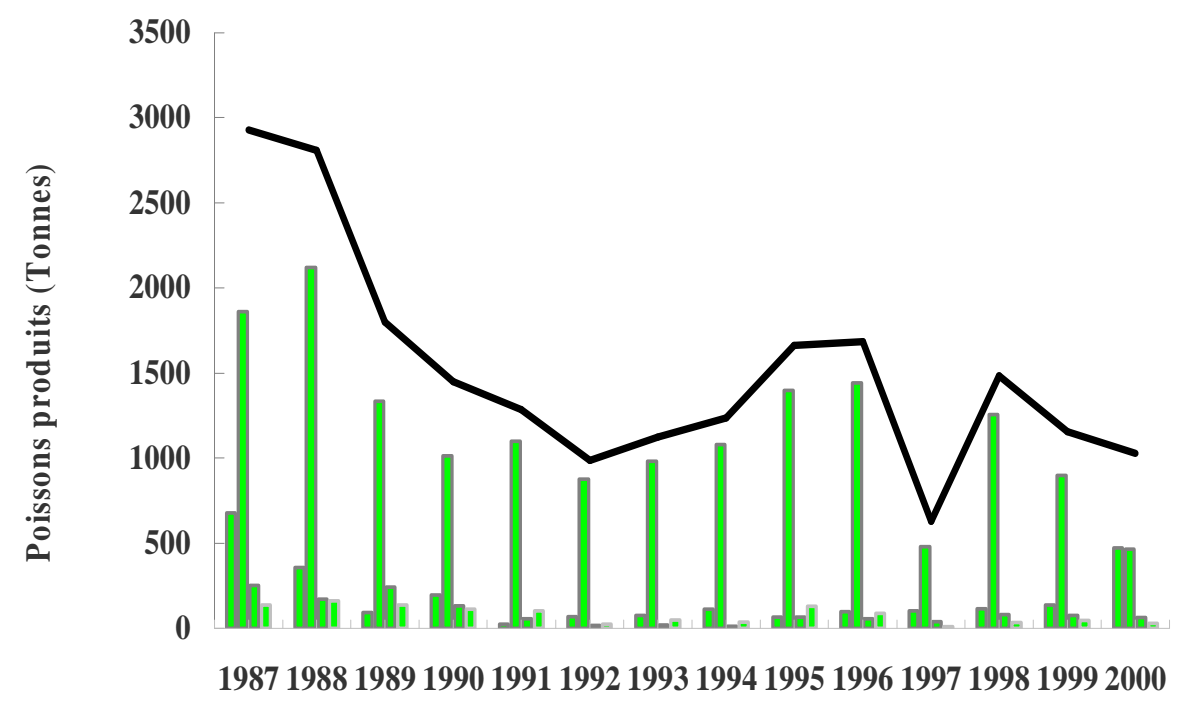

$\square$ Claroteidae

Clariidaee

$\square$ Mochokidae Années

Figure 10: Evolution de la production (en tonnes) de quatre principales familles de poissons-chats.

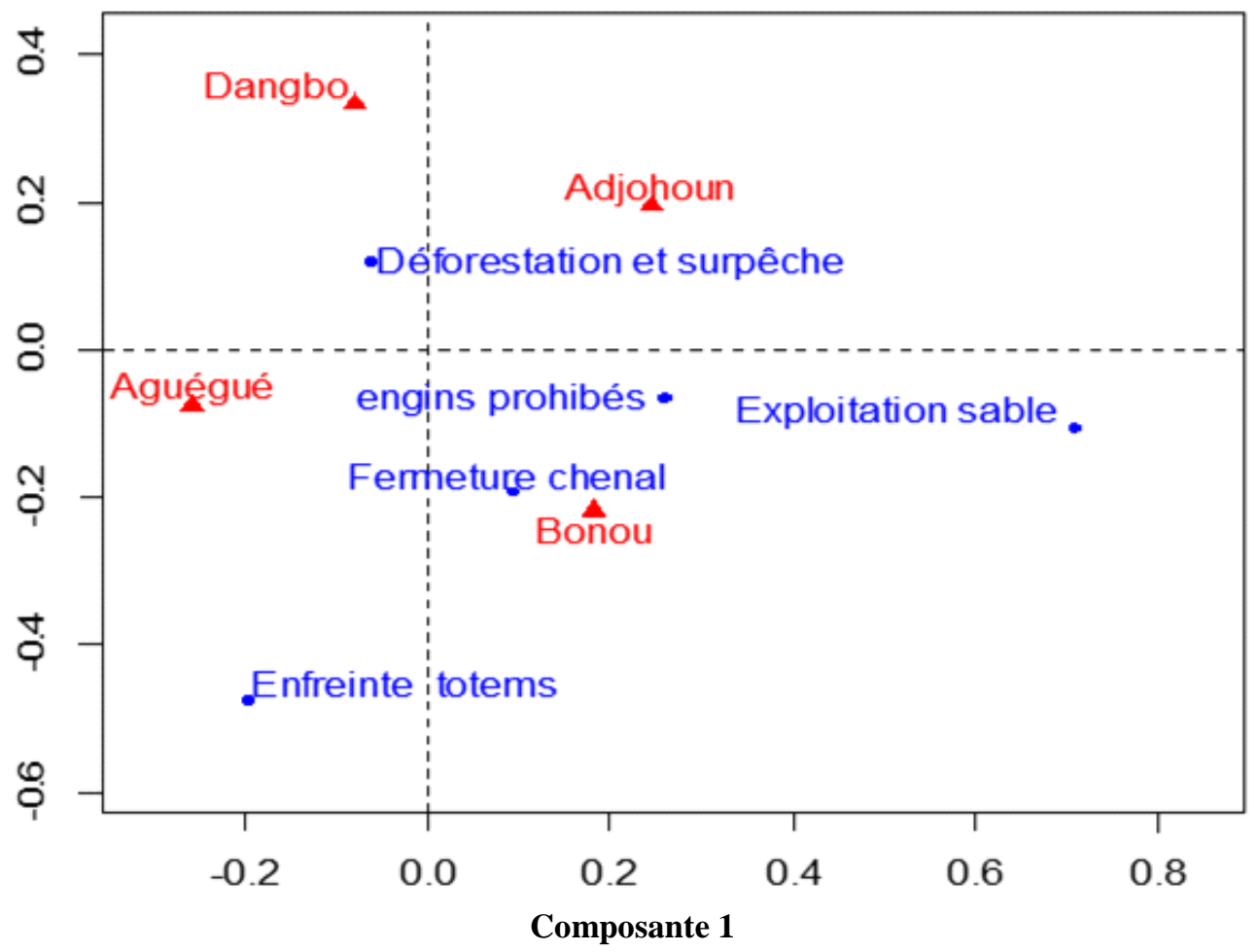

Figure 11: Actions favorisant l'ampleur des changements climatiques selon les pêcheurs suivant les 4 communes. 
Tableau 1: Caractéristiques socioprofessionnelles des acteurs de pêche par commune.

\begin{tabular}{cccccc}
\hline & & \multicolumn{4}{c}{$\begin{array}{c}\text { \% des répondants par commune } \\
\text { (BVO) }\end{array}$} \\
\hline Variables & Modalités & $\begin{array}{c}\text { Aguégué } \\
(\mathrm{n}=96)^{\mathbf{1}}\end{array}$ & $\begin{array}{c}\text { Dangbo } \\
(\mathrm{n}=44)\end{array}$ & $\begin{array}{c}\text { Adjohoun } \\
(\mathrm{n}=49)\end{array}$ & $\begin{array}{c}\text { Bonou } \\
(\mathrm{n}=77)\end{array}$ \\
& Féminin & 10,42 & 2,27 & 6,12 & 16,88 \\
Sexe & Masculin & 89,58 & 97,73 & 93,88 & 83,12 \\
& $<30$ ans & 4,17 & 0 & 0 & 0 \\
Age & Entre 30 et 60 ans & 81,25 & 86,36 & 91,84 & 89,61 \\
& $\geq 60$ ans & 14,58 & 13,64 & 8,16 & 10,39 \\
Origine & Autochtones & 100 & 100 & 100 & 100 \\
& migrants & 0 & 0 & 0 & 0 \\
Expérience dans & $<30$ ans & 54,17 & 54,55 & 59,18 & 63,64 \\
la pêche & Entre 30 et 60 ans & 45,83 & 45,45 & 40,82 & 36,36 \\
& $\geq 50$ ans & 0 & 0 & 0 & 0 \\
Niveau & Primaire & 14,58 & 11,36 & 6,12 & 12,99 \\
d'instruction & Secondaire & 0 & 4,55 & 8,16 & 2,6 \\
Contribution & analphabète & 85,42 & 84,09 & 85,71 & 84,42 \\
de la pêche & Très important & 13,54 & 15,91 & 20,41 & 16,88 \\
dans le revenu & Important & 23,96 & 25 & 18,37 & 29,87 \\
Du ménage & Pas important & 62,5 & 59,09 & 61,22 & 53,25 \\
\hline 1= nombre de personnes enquêtées $\quad$ BVO = basse vallée de l'Ouémé. & &
\end{tabular}

Tableau 2: Relation entre variables explicatives et perception des pêcheurs sur les changements climatiques.

\begin{tabular}{lccccc}
\hline Paramètres & DDL & Valeur estimée & Erreur type & Khi-2 de Wald & Pr> Khi-2 \\
\hline Intercept & 1 & $-6,7163$ & 2,1206 & 10,0313 & 0,0015 \\
Niveau d'instruction & 1 & $-0,00928$ & 0,2644 & 0,0012 & 0,9720 \\
Sexe & 1 & 5,0931 & 0,7020 & 52,6405 & $<0001 * * *$ \\
Age & 1 & 0,00964 & 0,0653 & 0,0218 & 0,8826 \\
Expérience & 1 & $-0,0269$ & 0,0905 & 0,0883 & 0,7663 \\
Religion & 1 & $-0,5593$ & 0,3031 & 3,4059 & 0,0650 \\
\hline \multicolumn{2}{c}{$* *=$ significatif au seuil de 5\%; • significatif au seuil de 10\%. }
\end{tabular}

\section{DISCUSSION}

Les pêcheurs de la basse vallée de l'Ouémé ont une bonne connaissance des changements climatiques. En effet, pour ces derniers, les changements climatiques se manifestent à travers la mauvaise répartition des pluies et leurs irrégularités. La chaleur intense, les inondations, les grandes poches de sècheresse (tarissement du fleuve par endroit notamment dans sa partie amont) et des grands bouleversements dans le calendrier des campagnes de pêche sur la durée sont aussi signalés. De nombreuses études menées dans le monde et en Afrique sont parvenues à des résultats similaires (Adamou et al., 2015; Vissin, 2013; Macharia et al., 2012; Mustapha et al., 2012). Les changements climatiques majeurs signalés par les pêcheurs sont le 
retard de pluies et l'augmentation de la température de l'eau. Traoré et al. (2002) et Hassan et Nhemechena (2008) ont noté que dans la Sous-Région Ouest africaine, les paysans ont perçu les changements climatiques à travers l'élévation des températures à long terme et à la diminution des précipitations, à des changements prononcés de la synchronisation des pluies, à la récurrence des sécheresses et au tarissement des cours d'eau autrefois pérennes pendant la saison sèche. Poirel et al. (2008) ont indiqué que la hausse de la température de l'eau est corrélable avec l'augmentation de la température de l'air. Le retard des pluies combiné à la diminution du nombre de jours de pluie et l'arrivée des eaux de pluie de l'Ouémé supérieur à certaines périodes de l'année permet de comprendre les nombreux cas d'inondation signalés dans la zone. En effet, la basse vallée est intégrée à l'Ouémé inférieur qui représente le réservoir de l'Ouémé supérieur et collecte toutes les eaux de l'amont du fleuve vers son delta puis vers la basse vallée de l'Ouémé (Lalèyè et al., 2004).

Au regard de la concordance entre perception des pêcheurs et les résultats d'analyse des données hydroclimatiques, il convient de noter que les communautés locales ont une bonne connaissance des événements climatiques. Tout comme les pêcheurs, Villeneuve, (2005) a noté que les changements climatiques ont accentué la chaleur depuis 1985 et ont entrainé la perte de biodiversité et la modification de la productivité.

Le sexe et la religion sont des facteurs déterminants dans la perception des changements climatiques. En effet, Oyekalé et Oladélé (2012) ont montré que dans les communautés rurales africaines, les hommes percevaient mieux les changements climatiques, probablement du fait de leur contact régulier avec le monde extérieur et leur meilleur accès aux sources d'information telle que la radio. Toutefois, cela peut être aussi lié au fait que les activités (agriculture, pêche) plus affectées durement par les changements climatiques sont quasi exclusivement exercées par les hommes dans cette communauté ; alors même que les effets des changements climatiques sur le petit commerce auquel s'adonnent les femmes ne peuvent être ressentis qu'à long terme. Gangle et al. (2011) indiquent que les changements climatiques sont perçus et compris de façons diverses par les populations africaines selon leurs caractéristiques socioculturelles.

Dans une certaine mesure, l'âge aussi est un facteur déterminant dans la perception climatique puisque ce sont les pêcheurs d'au moins 20 ans de pratique de pêche qui sont enquêtés. Sanchez-Cortés et Lazos Chavero (2011) ont aussi mis l'accent sur la capacité des personnes âgées à recourir au calendrier ethno climatique afin de comparer les changements détectés dans la variabilité du climat. Oyekale et Oladele (2012) ont aussi précisé qu'avec l'âge, l'habileté à percevoir les changements climatiques augmentait.

Les pêcheurs ont noté que les changements climatiques ont des incidences graves sur la pêche, tout comme Lalèyè et al. (2007) qui avaient signalé la baisse de rendement des principaux engins de pêche: $2,2 \mathrm{~kg}$ de poissons par jour de pêche pour le filet maillants, $3,5 \mathrm{~kg}$ pour le filet dobah et 1,4 $\mathrm{kg}$ pour le filet épervier contre respectivement $5,2 \mathrm{~kg} ; 4,3 \mathrm{~kg}$ et $1,6 \mathrm{~kg}$ obtenus dans la vallée de l'Ouémé au cours des années 196869.Villeneuve (2005) avait noté aussi que les changements climatiques ont des impacts sur l'ensemble des activités humaines. Dans le même ordre d'idée, Touré (2010) avait fait observer que dans le delta central du fleuve Niger, la baisse pluviométrique s'est accompagnée d'une baisse considérable de la production halieutique. Gillooly et al. (2001); et Wolter (2007) avaient indiqué que la 
température de l'eau influence le métabolisme des poissons, leur reproduction, leur développement et leur croissance ou encore leur comportement. Un réchauffement de l'eau induit une augmentation de l'activité métabolique des poissons jusqu'à ce que la température létale soit atteinte. L’influence des changements climatiques a été aussi relevée avec d'autres spéculations comme l'igname (Loko et al., 2013), le karité (Ganglè et al., 2012), le sorgho (MacCarthy et Vlek, 2012), le riz (Nwalieji et Uzuegbunam, 2012), le niébé (Ajetomobi et Abiodun, 2010).

Les pêcheurs de la basse vallée de l'Ouémé ont relevé la prolifération des parasites, des plantes ichtyotoxiques voire la pollution des plans d'eaux comme effets d'entrainement des changements climatiques. Villanueva et al. (2004) abondant dans le même sens dans le lac Nokoué, avait indiqué que les aléas climatiques ont contribué à modifier la biodiversité des milieux aquatiques tropicaux et ont indirectement accélérer leur dégradation.

Les pêcheurs ont aussi noté les fluctuations assez sensibles du niveau d'eau dans les différents lits du fleuve Ouémé dues à la succession des périodes d'inondation et d'étiage. Or, une modification du régime hydrologique a des conséquences sur la biodiversité aquatique. Tetzlaff et al. (2008) notent que les déplacements migratoires des espèces sont également sensibles aux variations du régime hydrologique des cours d'eau. En effet, les poissons sélectionnent généralement leurs habitats en fonction du régime des débits, de la vitesse du courant ou $\mathrm{du}$ volume d'eau disponible qui peuvent affecter leur croissance ou leur survie (Crozier et Zabel, 2006). Globalement, la baisse des rendements est la principale implication des changements climatiques sur la pêche selon les pêcheurs de la basse vallée de l'Ouémé, même si les actions favorables aux changements climatiques sont perçues avec quelques nuances d'une commune à une autre.

\section{Conclusion}

$\mathrm{Au}$ terme de cette étude, le constat général montre que les pêcheurs de la basse vallée de l'Ouémé sont conscients de la dégradation des facteurs hydroclimatiques dans leur cadre de vie, et son impact défavorable sur leurs activités de pêche. Leurs perceptions sont en adéquation avec la tendance évolutive de la température, de la pluviométrie et des statistiques de pêche dans la basse vallée de l'Ouémé, marquée par une variation sensible de la température, de la pluviométrie des lames d'eau écoulées (19712013) et des captures de pêche. Ces acteurs de pêche ont aussi une bonne idée des causes des variabilités climatiques. Toutefois, il convient de documenter les stratégies d'adaptation face aux effets néfastes des changements climatiques dans ce milieu avant de tirer des conclusions plus indiquées pour la prise de décision au profit de l'aménagement de cet écosystème.

\section{CONFLIT D'INTERETS}

Les auteurs déclarent qu'aucun intérêt n'est en compétition dans cet article.

\section{CONTRIBUTIONS DES AUTEURS}

Les auteurs ci-dessous ont participé de diverses manières à la conception de cet article. AHA est l'instigateur principal. EWV et SAM ont apporté un appui technique et ont lu le manuscrit. LHZ a lu et corrigé le manuscrit. PAL a dirigé et supervisé le travail.

\section{REMERCIEMENTS}

Les auteurs adressent leur remerciement au Projet de Productivité Agricole en Afrique de l'Ouest (PPAAO) du Programme Cadre d'Appui à la diversification agricole (ProCAD) du Ministère de 
l'Agriculture et de la Pêche (MAEP) au Bénin (Afrique de l'Ouest).

\section{REFERENCES}

Adamou MM, Alhou B, Nazoumou Y, Alloke G. 2015. Impacts des facteurs climatiques et anthropiques sur les ressources et la qualité des eaux de la mare de Tabalak. Int. J. Biol. Chem. Sci., 9(3): 1665-1677.

Ajetomobi J, Abiodun A. 2010. Climate change impacts on cowpea productivity in Nigeria. African Journal of Food, Agriculture, Nutrition and Development, 10(3): 2258-2217.

Assogbadjo AE, Glèlè Kakaï R, Edon S, Kyndt T, Sinsin B. 2011. Natural variation in fruit Characteristics seed germination and seedling growth of Adansonia digitata L. in Benin. New Forests, 41: 113-125.

Bowerman BL, O'connell RT. 1993. Forescasting and Time Series: An Applied Approach ( $3^{\text {rd }}$ Edn). Duxbury Press: USA; $672 \mathrm{p}$.

Brou YT, Akindès F, Bigot S. 2005. La variabilité climatique en Côte d'Ivoire: entre perceptions sociales et réponses agricoles. Cahiers Agricultures, 14(6): 533-540.

Crozier LG, Zabel RW. 2006. Climate impacts at multiple scales: evidence for differential population responses in juvenile Chinook salmon. Journal of Animal Ecology, 75: 1100-1109.

Dagnelie P. 1998. Statistiques Théorique et Appliquée: Inférence Statistique à une et deux Dimensions (Tome 2). De Boeck et Larcier: Paris-Bruxelles, France, Belgique ; 659p.

DP (Direction des pêches). 2005. Statistiques des pêches continentales (1987-2005) au Bénin. Ministère du Développement Rural et de l'action coopérative. Projet Pêche Lagunaire, 11: p 46.
Gnanglè PC, Glèlè Kakaï RL, Assogbadjo A, Vodounnon S, Yabi Afouda J, Sokpon N. 2011. Tendances climatiques passées, modélisations, perceptions et adaptations locales au Bénin. Climatologie, 8.

Gnanglè PC, Egah J, Baco MN, Gbémavo CDSJ, Glèlè Kakaï RL, Sokpon N. 2012. Perceptions locales du changement climatique et mesures d'adaptation dans la gestion des parcs à karité au NordBénin. Int. J. Biol. Chem. Sci., 6(1): 136149.

Gillooly JF, Brown JH, West GB, Savage Van M, Charnov EL. 2001. Effects of size and temperature on metabolic rate. Science., 293: 2248-2251.

Gbaguidi AA, Faouziath S, Orobiyi A, Akouegninou BA, Dansi A. 2015. Connaissances endogènes et perceptions paysannes de l'impact des changements climatiques sur la production et la diversité du Niébé (Vigna unguiculata (L.) Walp.) et du Voandzou (Vigna subterranean (L) Verde.) au Bénin. Int. J. Biol. Chem. Sci., 9(5): 2520-2541.

Hassan R, Nhemachena C. 2008. Determinants of African farmer's strategies for adapting to climate change: multinomial choice analysis. AfJARE, 2(1): 83-104.

INRAB. 1995. Fiches Techniques sur les Sols et les Essences Forestières. INRAB: Cotonou, Bénin; 68p.

Lalèyè AP, Chikou A, Philippart J-C, Teugels G, Vandewalle P. 2004. Étude de la diversité ichtyologique du bassin du fleuve Ouémé au Bénin (Afrique de l'Ouest). Cybium., 28(4): 329-339.

Lalèyè AP, Ezin A, Vandewalle p, Phillippart J-C, Teugels GG. 2007. Caractéristiques de la pêche dans le fleuve Ouémé au Bénin (Afrique de l'Ouest). J. Afrotrop. Zool., Special issue: 137-148.

Loko YL, Dansi A, Agre AP, Akpa N, Dossou-Aminou I, Assogba P, Dansi M, 
Akpagana K, Sanni A. 2013. Perceptions paysannes et impacts des Changements Climatiques sur la production et la diversité variétale de l'igname dans la zone aride du Nord-Ouest du Bénin. Int. J. Biol. Chem. Sci., 7(2): 672-695.

Macharia PN, Thuranira EG, Nganga LW, Lugadiru J, Wakori S. 2012. Perceptions and adaptation to climate change and variability by immigrants' farmers in semiarid region of Kenya. African Crop Science Journal, 20(1): 287-296.

Mustapha SB, Sanda AH, Shehu H. 2012. Farmer's of perception of climate change in central agricultural zone of Borno State. Nigeria. Journal of Environment and Earth Science, 2(11): 21-27.

MacCarthy DS, Vlek PLG. 2012. Impact of climate change on sorghum production under different nutrient and crop residue management in semi-arid region of Ghana: a modeling perspective. African Crop Science Journal, 20(2): 243-259.

Nwalieji HU, Uzuegbunam CO. 2010. Effect of climate change on rice production in Anambra State, Nigeria. Journal of Agriculture Extension, 16(2): 81-91.

O'Brien K, Wolf J. 2010. A values-based approach to vulnerability and adaptation to climate change. Wiler Interdisciplinary Reviews: Climate Change, 1: 232-242.

Ogouwalé E, Boko M. 2007: Evolution des climats et risques agricoles dans le Bénin méridional et central. Climat et Développement, 3: 34-44

Oyakale AS, Oladele OI. 2012. Determinants of climate change and adaptation among cocoa farmer in southwest Nigeria. Journal of Food, Agricuture and Environement, 10(3-4): 1562-1567.

PANA-Bénin. 2008: Rapport du Programme d'Action National d'Adaptation aux changements climatiques du Bénin. Cotonou, 58p.
Patt A GM, Tadross P, Nussbaumer K, Assante M, Mertzger J, Rafael A, Goujon G, Brundrit G. 2010. Estimating least-developed countries' vulnerability to climate-related extreme events over the next 50 years. Proceeding of the National Academy of Sciences, 107: 1333-1337.

Poirel A, Lauters F, Desaint B. 2008. 19772006: Trente années de mesures des températures de l'eau dans le Bassin du Rhone. Hydroécol. Appl., 16: 191-213.

Sanchez-Cortés MS, Lazos Chavero E. 2011. Indigenous perception of Changes in climate variability and its relationship with agriculture in a Zoque Community of Chiapas, Mexico. Climatic Change, 107: 363-389.

Sonneveld BGJS, Keyzer MA, Adegbola P, Pande S. 2012. The Impact of Climate change on Crop Production in West Africa: An Assessment for the Oueme River Basin in Benin. Water Resource Management, 26: 553-579.

Tetfzlaff D, Gibbins C, Bacon PJ, Youngson AF, Soulsby C. 2008. Influence of hydrological regimes on the prespawning entry of Atlantic Salmon (Salmo Salar L.) into an upland river. River Research and Applications, 24: 528-542.

Traoré AF, Diallo ML, Bamba Z, Mara F. 2002. Communication initiale de la Guinée sur la Convention Cadre des Nations Unies sur les Changements Climatiques. Projet FEM/PNUDGUI/97/G33, Conakry, 187.

Toure A. 2006. Sédentarisation et adaptation des pêcheurs du delta central du Niger: cas des communes de Mopti et Konna. Université de Bamako, République du Mali, 58p.

Villanueva C-M. 2004. Biodiversité et relations trophiques dans quelques 
milieux estuariens et lagunaires de l'Afrique de l'Ouest: Adaptation aux pressions environnementales. $\mathrm{PhD}$ Thesis, Institut National Polytechnique de Toulouse.

Villeneuve C. 2005. Vivre les changements climatiques. Conférence au CEGEP de Ste Foy du 20 octobre 2005. Université du Québec à Chicoutimi, Département des sciences fondamentales, 42p.

Vissin EW. 2013. Sensibilité de la pluviométrie à la TSO en Août dans le Bénin méridional et central (Golfe de Guinée). Climat et Développement., 15: 58-66.

Vodonou JM, Gohoungossou A, Sintondji L, Lawin E, et Zannou A. 2011.
Contribution du Projet Ouémé. 2025 à l'étude de la dynamique et de la disponibilité des ressources en eau du bassin de l'Ouémé. Changements Environnemtaux, 3: 30-46.

Wolter C. 2007. Temperature influence on the fish assemblage structure in a large lowland river, the lower Oder River, Germany. Ecology of Freshwater. Fish., 16: 493-503.

Yabi I, Boko, Sinsin B. et Afouda.2009: Variabilité et production de l'anacardier dans le centre du Bénin. Climat et Développement, 7: 40-50. 